\title{
RELACIÓN ENTRE LA SATISFACCIÓN CON LA IMAGEN CORPORAL, AUTOCONCEPTO FÍSICO, ÍNDICE DE MASA CORPORAL Y FACTORES SOCIOCULTURALES EN MUJERES ADOLESCENTES COSTARRICENSES
}

\author{
${ }^{1 y 2}$ García Fernández Laura y ${ }^{1 y 3}$ Garita Azofeifa Elmer \\ ${ }^{1}$ Escuela Ciencias del Deporte, Universidad Nacional \\ ${ }^{2}$ Escuela de Nutrición, Universidad de Costa Rica \\ ${ }^{3}$ Escuela de Educación Física y Deportes, Universidad de Costa Rica. \\ E-mail: laugarfer@gmail.com
}

\begin{abstract}
Resumen
García Fernández, L. y Garita Azofeifa, E. (2007). Relación entre la satisfacción con la imagen corporal, autoconcepto físico, índice de masa corporal y factores socioculturales en mujeres adolescentes costarricenses. Revista de Ciencias del Ejercicio y la Salud, 5(1), 9-18. El propósito del estudio fue analizar la relación entre la satisfacción con la imagen corporal (IC) con el autoconcepto físico (AF), el índice de masa corporal (IMC) y factores sociales, en una muestra de 99 mujeres $\left(15,85 \pm 0,98\right.$ años de edad e IMC de 21,62 $\left.\pm 3,09 \mathrm{~kg} / \mathrm{m}^{2}\right)$. Se utilizó: la escala de Stunkard y cols. (1983) para establecer la satisfacción con la IC; el cuestionario de Marsh y Southerlan (1994) para medir el AF general y sus dimensiones y otro cuestionario elaborado para esta investigación, donde se recopiló datos personales, sociales e indicadores de la composición corporal para el cálculo del IMC. Los resultados mostraron que el grupo de adolescentes satisfechas con su IC reportaron un puntaje promedio mayor en apariencia física pero menor en el AF general en comparación con las insatisfechas. Se encontró relación significativamente negativa entre IMC y la apariencia física. Por lo tanto se concluye que la muestra en estudio basa la satisfacción de su IC en relación con indicadores corporales como el peso, de manera que a menor peso más satisfechas están con su apariencia física e IC, por lo tanto, se le debe dar una orientación adecuada a esta muestra de sujetos para evitar que puedan caer en algún tipo de trastorno de alimentación, como método drástico para la reducción de peso.
\end{abstract}

PALABRAS CLAVES: adolescencia, imagen corporal, satisfacción, autoconcepto físico, factores socioculturales.

\section{INTRODUCCIÓN}

La adolescencia es una de las etapas del desarrollo humano que plantea más retos, puesto que el crecimiento físico que se presenta está acompañado de un desarrollo emocional e intelectual rápido. La capacidad de los adolescentes para el pensamiento abstracto, por contraposición a los patrones de pensamiento concreto de la infancia, les permite realizar tareas propias de este período, como es el establecimiento de su imagen corporal (Mahan y Escott-Stump, 2000). La imagen corporal (IC) es un "constructo psicológico complejo, que se refiere a cómo la autopercepción del cuerpo/apariencia genera una representación mental, compuesta por un esquema corporal perceptivo y así como las emociones, pensamientos y conductas asociadas" (Baile, 2000, p.5). Otra definición para este concepto es la que la precisa como la manera en que las personas se perciben a sí mismas y como piensan que son vistas por las demás personas (Cash y Pruzinsky, 1990, citado por Benninghoven, Eckhard, Mohr, Heberlein, Kunzendorf y Jantschek, 2006).

La IC está en constante cambio, modificándose por factores biológicos y circunstancias de la vida, acentuada por el placer o el dolor (Benninghoven, Eckhard, Mohr, Heberlein, Kunzendorf, y Jantschek, 2006). Este concepto es generalmente un constructo multidimensional que comprende componentes perceptuales, cognitivos, afectivos y del comportamiento. El 
componente perceptual incluye la conciencia de las dimensiones de su cuerpo y su medición por instrumentos que requiere que una persona evalúe el tamaño de todo su cuerpo o de algunas de sus partes. Mientras que los componentes cognitivos tienen que ver con las creencias sobre su propio cuerpo, como la satisfacción corporal (Bane y McAuley, 1998; Thompson, 1995, citado por Rowe, McDonald, Mahar y Raedeke, 2005).

Fallas (2001) indica que las personas que experimentan una gran multiplicidad de movimientos, desarrollan de forma más efectiva su IC, ya que el movimiento agudiza la percepción de ésta, por que promueve el hecho de conocer lo que se puede y no se puede realizar, de acuerdo a la habilidad de las diferentes cualidades físicas que se posee según la oportunidad de desarrollo, además de características corporales de cada individuo.

A parte del movimiento otros trasmisores y reforzadores de los ideales corporales según la teoría sociocultural, son los medios colectivos y otros recursos de presión sociocultural como la familia, los amigos y el ambiente (Levine y Harrison, 2004), que pueden muchas veces provocar alteraciones de la IC, tales como la distorsión del tamaño corporal y la insatisfacción corporal. La distorsión del tamaño corporal es un estimado erróneo en la percepción del tamaño del propio cuerpo de una persona, mientras que la insatisfacción corporal es la evaluación negativa que hace una persona de su cuerpo (Cash y Deagle, 1997). Rowe y cols., (2005), definen la insatisfacción corporal como la diferencia entre el tamaño corporal que una persona percibe y el que desea.

El efecto de esta influencia sociocultural fue demostrado en el 2002 por un metaanálisis realizado por Groesz, Levine y Murnen, en el que mostraron que las mujeres se sienten peor después de estar expuestas a imágenes de modelos femeninas delgadas, más que a otros tipos de imágenes.
Otro aspecto importante con el que se relaciona la IC es el papel que tiene en las relaciones sociales. Davison y McCabe, (2006), describen que la IC puede ser importante diferentemente tanto para las relaciones del mismo sexo como con el sexo opuesto, debido a la diferencia cualitativa de la naturaleza de estas relaciones durante la adolescencia. Esto basado en la psicología social, la cual ha mostrado el impacto de la apariencia física de un individuo y cómo otros lo perciben e interactúan con el o ella, en comparación con sujetos no atractivos recibiendo evaluaciones negativas de sus semejantes y reduciendo el contacto social. (Langlois y Stephan, 1981, citado por Davison y McCabe, 2006).

Lin y Kulik (2002), sugieren que las mujeres jóvenes con novio, debe ser menos probable que se comparen con las modelos delgadas del medio y en consecuencia tengan menos insatisfacción corporal y alta autoestima en comparación con las que no tienen novio. De igual forma, Forbes, Jobe y Richarson (2006), examinaron la asociación entre tener novio y la satisfacción corporal y autoestima, encontrando que el tener novio se asoció con menor insatisfacción corporal en mujeres universitarias. Así mismo, Whitehead y Hoover (2000), basados en las indicaciones preliminares de que la IC juega un rol en las interacciones sociales de los adolescentes, sugieren que los muchachos y muchachas comúnmente expresan tener sobrepeso como una excusa por estar solos, sin pareja.

La insatisfacción corporal, ha sido relacionada con baja autoestima, depresión y con el impulso inicial en el comportamiento de desórdenes de la conducta alimenticia, como anorexia y bulimia nerviosa, especialmente en mujeres adolescentes o adultas jóvenes (Stice, 2001; Baile, Raich y Garrido, 2003; Polivy y Herman, 2002; Thompson, 2004), ya que para esta población la apariencia es más centrada en el autoconcepto y la evaluación de los demás (Grogan, 1999), y socioculturalmente la belleza ideal femenina es ser ultra 
delgada, la que es inalcanzable e insalubre (Halliwell y Dittmar, 2004).

En pacientes con desordenes alimenticios, su IC es diferente y puede cambiar con el tiempo o durante el tratamiento. Aunque algunos de estos sujetos cuyo peso es inferior a lo normal, se sientan "enormes", debido a que según investigaciones se ha documentado que todos los elementos de la IC están severamente disminuidos (Probst, Morris, Vandereycken, Van Coppenolle, 1996; Kerruish, Connor, Hunphris y cols., 2002). La grasa así como la musculatura corporal total se reduce marcadamente en el estado de bajo peso. Muchos pacientes temen específicamente la ganancia de peso que resulta en el desproporcional e inaceptable incremento en el circunferencia abdominal (Mayer y cols., 2005).

Los trastornos de alimentación se clasifican como enfermedades psiquiátricas, y se acompañan de complicaciones médicas importantes, morbilidad y tasas de mortalidad de ente un 5 y $15 \%$ (Mahan y Escott-Stump, 2000), por lo que representan un problema de consideración para la salud pública ya que afecta a un número considerable de mujeres de nuestra sociedad.

Dentro de los métodos que han sido usados para medir varios componentes de la IC, se incluyen cuestionarios, entrevistas, dibujos y técnicas de distorsión de imagen (Rowe y cols., 2005).

En los últimos años se ha incrementado el uso de las siluetas o figuras para la medición del tamaño corporal, forma, peso y satisfacción con la apariencia. Cada escala tiene en común la presentación de un número discreto de siluetas normalmente entre 5 y 12 . Con frecuencia los sujetos son indicados a seleccionar la figura que representa su actual talla y luego la talla que consideran ideal. La diferencia entre los datos es llamado el índice de discrepancia y es considerado para representar la insatisfacción corporal (Gardner y cols., 1998; Rowe y cols., 2005).
Stunkard, Sorenson y Schlusinger, 1983 (citados por Gardner y cols., 1998), desarrollaron una serie ampliamente usada de nueve figuras esquemáticas tanto masculinas como femeninas, ubicadas desde muy delgado hasta muy obeso (ver figura 1). Así mismo, otros investigadores han desarrollado figuras similares para una variedad de poblaciones incluyendo desde bebés hasta adultos mayores (Rand y Wright, 2000).

Figura 1. Escala de Stunkard y cols., (1983) (cit. en Baile, Raich, y Garrido, 2003)
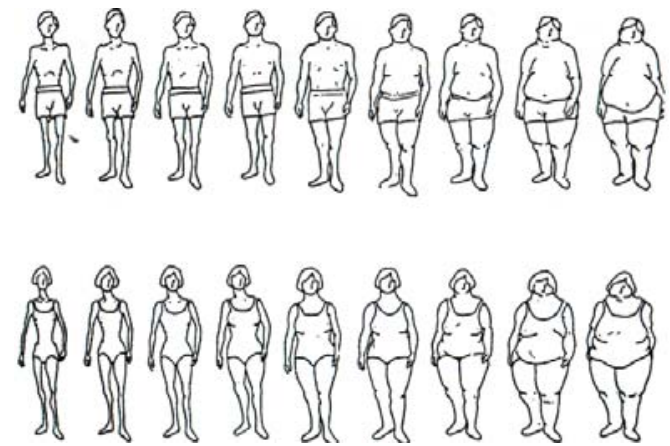

Para la medición de la composición corporal además de la utilización de escalas con siluetas también se emplea la antropometría, que se refiere al estudio del tamaño, la forma, la proporción, la maduración y el funcionamiento general del cuerpo (Rodríguez y Ulate, 1999), la cual tiene una importancia especial durante todas las etapas de desarrollo y es bastante marcada durante la adolescencia, porque permite vigilar y evaluar los cambios mediados por hormonas en el crecimiento y la maduración en este periodo, además proporciona indicadores del estado nutricional y el riesgo para la salud, y puede aportar el diagnóstico de la obesidad (OMS, 1993). Uno de los índices antropométricos que se utilizan para tal fin, es el Índice de Masa Corporal (IMC), que se refiere a la razón entre la masa corporal en kilogramos, dividido por la talla (estatura) en metros elevada al cuadrado 
$\left(\mathrm{IMC}=\left(\mathrm{kg} / \mathrm{m}^{2}\right) \quad(\right.$ Mahan $\mathrm{y}$ Escott-Stump, 2000).

El objetivo principal de este estudio es analizar si existe relación entre la satisfacción de la IC, el AF, el IMC y factores socioculturales, como la influencia de la televisión y el tener o no novio, en una población de mujeres adolescentes. Con el propósito de descubrir las relaciones más significativas entre estas variables, las cuales pueden afectar de manera negativa la IC y de esta forma, establecer lineamientos de trabajo que permitan prevenir que esta insatisfacción, se oriente a conductas que incidan en el deterioro del bienestar de la salud física y mental de las adolescentes, por el hecho de calzar con un patrón estético sin importar el precio que se tenga que pagar para alcanzarlo.

\section{METODOLOGIA}

\section{Sujetos}

La muestra seleccionada por conveniencia para ésta investigación, estuvo conformada por 99 alumnas de nivel económico medio y medio-alto, pertenecientes al Colegio Seráfico San Francisco; centro semiautónomo de Educación Secundaria ubicado en la región de Cartago. El rango de edad de los sujetos es de 15 a 18 años, con una edad promedio y desviación estándar de $15.85 \pm 0.98$ años. El peso promedio y desviación estándar de estos sujetos es de $54.72 \pm 8.98 \mathrm{~kg}$ y la talla fue de $157.98 \pm 11.63 \mathrm{~cm}$, por lo que presentan un IMC promedio de $21,62 \pm 3,09 \mathrm{~kg} / \mathrm{m}^{2}$.

\section{Instrumentos y Materiales}

Para la medición de la satisfacción de la IC se utilizó la escala de Stunkard y cols. (1983; cit. en Baile, Raich, y Garrido, 2003), que consiste en serie de nueve siluetas de mujeres con un rango desde muy delgadas hasta muy obesa.
Los factores socioculturales relacionados con la satisfacción de la IC fueron: la influencia de la televisión y las relaciones personales. En la primera se analizó la cantidad de horas de televisión que el sujeto observa diariamente, mediante la siguiente escala: $1=$ menos de 1 hora, $2=$ de 1 a 2 horas y $3=$ más de 3 horas. El segundo factor se relacionó con el hecho de tener o no novio en el momento de la recolección de los datos.

Se incluyó un cuestionario de realización específica para este estudio, que incluía una sección de datos personales del sujeto (edad y nivel académico) y otra para recopilar el peso y la talla autopercibida. Se incluyó la pregunta: ¿cuando usted se mira al espejo, con respecto a su imagen se siente de forma general: a) satisfecha o b) insatisfecha.

El AF se estimó mediante el cuestionario de autoconcepto físico de Marsh y Southerland (1994; cit. en Fallas, 2001), compuesto por 50 ítems, los cuales se evalúan tomando como base una escala de Likert de seis opciones, donde $6=$ totalmente de acuerdo, hasta y $1=$ totalmente en desacuerdo. El cuestionario presenta 6 dimensiones según su estructura factorial: concepto de apariencia física, de habilidad física, de fuerza, de equilibrio, de flexibilidad y de resistencia y reporta una consistencia interna de $\mathrm{r}=0.70$ (Fallas, 2001). Para efectos del presente estudio se utilizó el puntaje general obtenido y el correspondiente a la dimensión apariencia física.

Para calcular el IMC se recopiló las mediciones de peso y talla corporal por triplicado para cada sujeto (en una lista en general para todos los sujetos), siguiendo los protocolos estandarizados para la evaluación de cada una de estas variables. Para recopilar el peso se utilizó una balanza digital calibrada, marca Tanita modelo BF-680, con una sensibilidad de $0,1 \mathrm{~kg}$, y un tallímetro 
marca SECA de 2 metros de longitud para reportar la estatura.

\section{Procedimiento}

Inicialmente se estableció contacto con la institución para solicitar el permiso pertinente para desarrollar el estudio. Los datos se recopilaron durante la mañana y la tarde de dos días consecutivos dentro del horario escolar, en el transcurso de las lecciones de educación física. El espacio físico que se utilizó fue oficina de dicho departamento, con el fin de evitar que los sujetos se dispersaran y así aclarar las dudas de forma general.

A cada grupo seleccionado para la muestra, primero se les explicó el objetivo de la investigación y se les motivo a participar voluntariamente de la misma, reafirmando que los datos que iban a suministrar eran $100 \%$ confidenciales; también se aclaró el rango de edad para poder participar. Posteriormente, de manera grupal se les entregó primero el cuestionario de autoconcepto físico de Marhs y Southerland (1994), conforme terminaron de llenar este cuestionario se les entrego el siguiente, que incluía datos personales (edad, nivel académico), preguntas relacionadas con los factores socioculturales y con peso y talla autopercibidos, además contenía la escala de Stunkard y col. (1983), que se utilizó en la formulación de 3 preguntas: 1. ¿Cúal de estas figuras es usted actualmente? y 2. ¿Cuál de estas figuras le gustaría ser?

Ambos formularios estuvieron enumerados de modo que cuando el sujeto entregaba el primero, recogía el siguiente con el número asignado desde el inicio, por lo cual se le solicitó a los sujetos que recordaran o si era necesario que apuntaran este número.

Durante la aplicación de los dos cuestionarios se procuró que las respuestas fueran personales e individualizadas, evitando las influencias por parte de las compañeras cercanas. El tiempo total para la resolución de ambos test, fue de aproximadamente 15 minutos.

Una vez concluido los dos cuestionarios, el grupo se retiró con el profesor a ejecutar el trabajo correspondiente para ese día, posteriormente se llamó a las estudiantes en tríos para evaluarles el peso y la talla, utilizando los instrumentos descritos anteriormente. Se realizaron mediciones por triplicado, para obtener una medición precisa y exacta de dichos indicadores. Esta evaluación se realizó de manera individual entre el antropometrista y el sujeto, mientras tanto los otros dos sujetos aguardaban afuera de la oficina hasta que saliera su compañera. Antes de realizar la evaluación antropométrica se solicitó el número asignado para poder recopilar la información en una tabla de datos enumerada con el número total de la muestra de la población.

Seguidamente a la recolección de los datos se tabulo los mismos, creando una base de datos en el paquete estadístico SPSS versión 8.0 para realizar el análisis estadístico descriptivo e inferencial. También se utilizó el programa Microsoft Office Excel 2003, para la elaboración de cuadros y gráficos.

\section{Análisis Estadístico}

$\begin{array}{lccr}\text { Los datos se analizaron } & \text { con } \\ \text { estadística } & \text { descriptiva por medio } & \text { de } \\ \text { promedios, } & \text { desviaciones estándar } & y \\ \text { frecuencias. } & \text { Con respecto al análisis }\end{array}$
inferencial se aplicó la prueba t-Student para mediciones repetidas, para analizar las mediciones de: peso, talla e IMC percibido y evaluado antropométricamente. La t-Student de grupos independientes, se utilizó para analizar el AF, apariencia física para el grupo de adolescentes satisfechas e insatisfechas con su la IC. La prueba Chi cuadrada, se usó para relacionar la satisfacción de la IC con el $\mathrm{AF}$, apariencia física, horas de televisión, tener o no novio y con el IMC evaluado 
antropométricamente. La correlación de Pearson, se empleó para determinar si existe relación significativa entre el IMC y el AF, la apariencia física, la cantidad de horas de televisión al día o el tener o no novio. Se tomó como base para los cálculos e interpretación el texto de Moncada (2005).

\section{RESULTADOS}

A continuación se detallan los resultados obtenidos de acuerdo al análisis estadístico que se estableció anteriormente.

Tabla $\mathbf{N}^{\circ} 1$. Relación entre talla, peso e índice de masa corporal percibida y evaluada antropométricamente

\begin{tabular}{ccc}
\hline \multicolumn{1}{c}{ Relación } & t & p \\
\hline Talla $(\mathrm{cm})$ evaluada y autopercibida & $-1,803$ & 0,075 \\
Peso(kg) evaluado y autopercibido & 2,448 & $0,016^{*}$ \\
\hline IMC $(\mathrm{kg} / \mathrm{m} 2)$ evaluado y autopercibido & 2,667 & $0,009^{*}$ \\
\hline${ }^{*} \mathrm{p}<0.05$ & &
\end{tabular}

Gráfico N ${ }^{0} 1$. Peso promedio percibido y evaluado antropométricamente

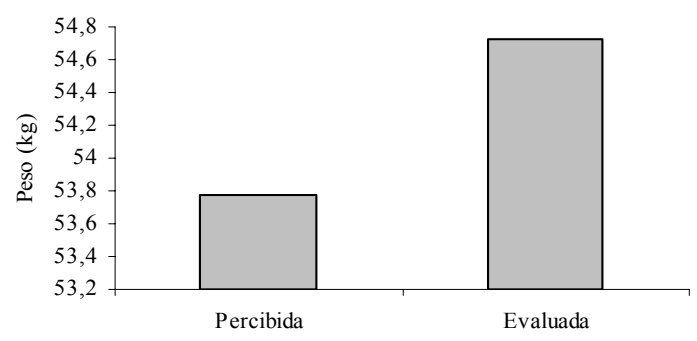

Gráfico $\mathrm{N}^{\circ} 2$. Índice de masa corporal promedio percibido y evaluado antropomé tricamente

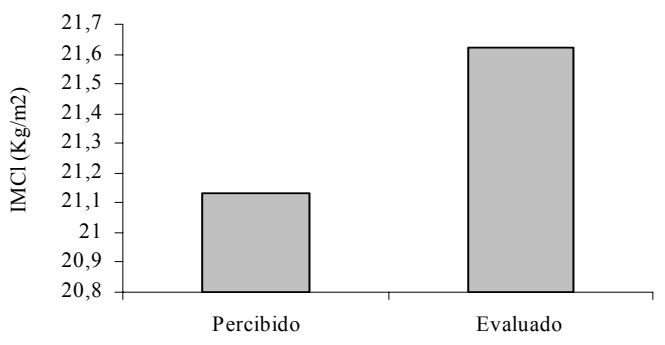

La tabla $\mathrm{N}^{\circ} 1$ muestra el análisis estadístico para peso, talla e IMC efectuado mediante una prueba de t-Student para mediciones repetidas. Esta prueba demostró diferencias significativas entre los promedios del peso percibido y el evaluado, siendo menor el promedio del peso percibido $(53,77 \mathrm{~kg}$ percibido y $54,72 \mathrm{~kg}$ evaluado $)$ como se observa en el gráfico 1 . También se encontró diferencias significativas entre el IMC percibido y el IMC evaluado, siendo mayor el promedio del IMC evaluado $(21,62$ $\left.\mathrm{kg} / \mathrm{m}^{2}\right)$ que el IMC percibido $\left(21,13 \mathrm{~kg} / \mathrm{m}^{2}\right)$, según se detalla en el gráfico 2 .

Mediante al análisis descriptivo de los datos, se encontró que un $77 \%$ de las adolescentes que participaron en este estudio indicaron que estaban satisfechas con su IC al preguntarles como se sentían cuando se miraban al espejo, pero al determinar la satisfacción mediante la escala de Stunkard y cols., sólo un $35,4 \%$ demostró estar satisfecha con su IC, ya que coincidió la figura que indicaba que eran en este momento y cual les gustaría ser.

Tabla $\mathbf{N}^{\circ} 2$. Relación de la satisfacción de la IC con la edad, los factores socioculturales y el IMC, y entre el IMC y las relaciones personales

\begin{tabular}{lcc}
\hline \multicolumn{1}{c}{ Relación } & $\chi^{2}$ & $\mathbf{p}$ \\
\hline Satisfacción IC*IMC & 16,264 & $0,000^{*}$ \\
Satisfacción IC*Horas de TV & 0,414 & 0,813 \\
Satisfacción IC*Novio & 1,500 & 0,221 \\
Satisfacción IC*Edad & 7,116 & 0,68 \\
Novio*IMC & 0,086 & 0,958 \\
\hline \multicolumn{2}{c}{$* 0.05$} &
\end{tabular}

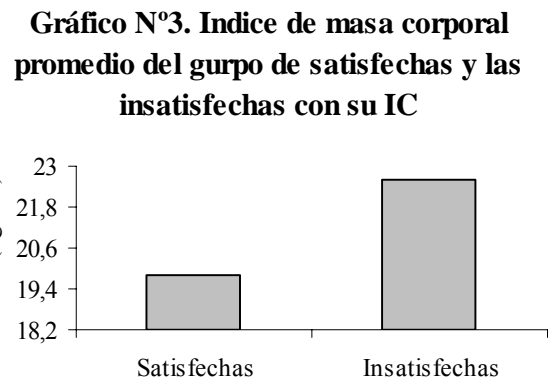


La satisfacción de la IC se analizó con respecto a las otras variables mediante una prueba de Chi Cuadrada (Tabla No2). Por medio de la aplicación de esta prueba se encontró que ni la edad, ni las horas de televisión diarias, ni el tener o no novio, marcan una influencia significativa en la satisfacción de la IC para esta población. Sin embargo es importante destacar que mediante el análisis de esta prueba se mostró que del $64.6 \%$ del total de adolescentes insatisfechas, un $33,3 \%$ observan la mayor cantidad de horas de televisión al día y que $41,4 \%$ de no tienen novio.

Esta prueba si demostró que existe diferencia significativa entre el grupo de las adolescentes satisfechas e insatisfechas con respecto a su IMC, es decir, el IMC es la única variable que influye en la satisfacción de la IC para la población de estudio. En el gráfico $\mathrm{N}^{\circ} 3$ se puede observa que las adolescentes satisfechas presentan un IMC promedio inferior que las insatisfechas $(19,8$ y $22,6 \mathrm{~kg} / \mathrm{m}^{2}$ respectivamente). Cabe mencionar que del total de adolescentes insatisfechas con su IC, un $42,4 \%$ presenta un peso normal de acuerdo a su talla.

También se utilizó una de prueba del Chi cuadrado para establecer la relación entre el IMC evaluado y el tener o no novio, ésta no estableció diferencia significativa entre ambas variables (tabla $\mathrm{N}^{\circ} 2$ ).

Tabla Nº3. Relación de la satisfacción de la IC con la apariencia física y el autoconcepto físico en general.

\begin{tabular}{lcc}
\hline \multicolumn{1}{c}{ Dimensión } & $\mathbf{t}$ & $\mathbf{p}$ \\
\hline Apariencia Física & 4,027 & $0,000^{*}$ \\
Autoconcepto físico general & $-0,532$ & 0,596 \\
\hline \multicolumn{2}{c}{$* \mathrm{p}<0.05$} &
\end{tabular}

Gráfico $\mathrm{N}^{\circ} 4$. Puntaje promedio de la apariencia física según satisfaccción de la IC

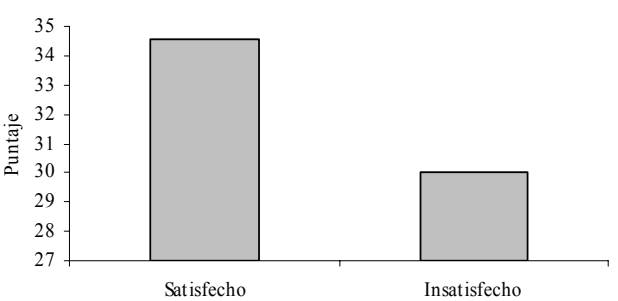

Gráfico $\mathrm{N}^{\circ} 5$. Puntaje promedio del autoconcepto físico según la satisfacción de la IC

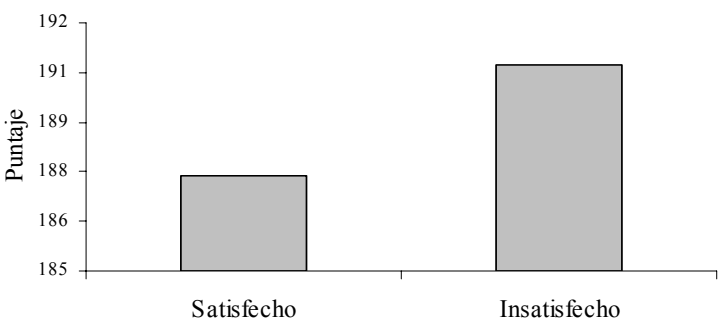

Los gráfico $\mathrm{N}^{\mathrm{o}} 4$ y $\mathrm{N}^{0} 5$, muestran los puntajes promedios para la apariencia física y el AF general, respectivamente, según la satisfacción de la IC. Se puede observar que las adolescentes satisfechas con su IC tienen en promedio una apariencia física mayor pero un AF menor que las insatisfechas; no obstante el análisis de t-Student de grupos independientes indicó que existe diferencia significativa entre la apariencia física y la satisfacción de la IC, pero entre esta y el AF general, según los datos de la Tabla N³.

\section{Tabla $N^{\circ} 4$. Correlaciones del índice de masa} corporal

\begin{tabular}{lcc}
\hline \multicolumn{1}{c}{ Relación } & $\mathbf{r}$ & $\mathbf{p}$ \\
\hline IMC*Apariencia Física & -0.337 & $0.001^{*}$ \\
IMC*Autoconcepto físico & -0.075 & 0.460 \\
IMC*Horas de televisión & 0.026 & 0.795 \\
\hline \multirow{2}{*}{$\mathrm{p}<0.05$} & &
\end{tabular}

Gráfico $\mathbf{N}^{\circ} 6$. Puntaje promedio de la apariencia física según la interpretación del índice de masa corporal

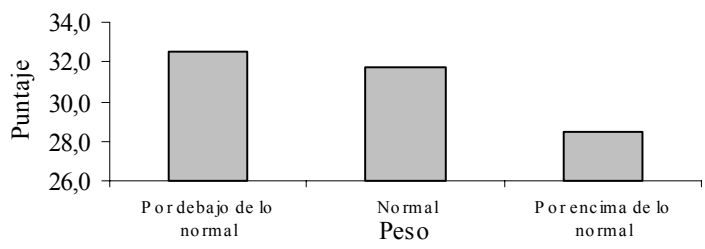


Con la aplicación de las correlaciones de Pearson entre IMC y apaciencia física, autoconpecto físico $\mathrm{u}$ horas de televisión, no se halló relación significativa entre IMC y el AF o las horas de TV, sin embargo, si se encontró una relación significativamente negativa entre el IMC y la apariencia física, es decir, que a menor IMC mayor apariencia física. Esta tendencia se observa en el gráfico $\mathrm{N}^{\circ} 6$, el cual demuestra que los sujetos que tienen un IMC por debajo de lo normal tienen una apariencia física promedio mayor que las que tienen un IMC normal o un IMC por encima de lo normal.

\section{DISCUSIÓN}

Es importe tener presente que la percepción de la IC no es estática y que especialmente en etapas de desarrollo como la adolescencia se modifica constantemente, como producto de los cambios que se generan a partir del desarrollo físico y psicológico, y también por las influencias y tendencias generadoras del medio ambiente en el que se desenvuelve el adolescente. Este estudio demostró que los sujetos presentan una alteración en la percepción de las dimensiones de su cuerpo, lo cual sugiere que el componente perceptual y cognitivo de la IC en estas adolescentes se encuentra alterado (Benninghoven y cols., 2006). Esto fundamentado como producto de la diferencia significativa encontrada entre el peso percibido y el evaluado mediante instrumentos antropométricos; donde de acuerdo a los promedios registrados para cada método de medición, se registró que ésta población de estudio no presenta una tendencia orientada a la sobreestimación del peso corporal sino más bien a la subestimación, es decir creen tener un peso menor al que en realidad poseen.

De acuerdo a los datos analizados la mayor cantidad de la población en estudio está insatisfecha con su IC, ya que se encontró diferencia entre el tamaño corporal que ellas creen tener y el que desean. De ésta cantidad total, más de la mitad tienen un peso adecuado para su talla, por lo cual la opción de reducir peso no es un fundamento válido ni saludable, ya que se encuentran dentro del parámetro normal. Esta tendencia orientada a la reducción corrobora lo que Baile y Garrido (s.f) señalan en su estudio, que en nuestra sociedad actual el estereotipo de una mujer muy delgada es válido y adecuado, el cual es expresado a éxito, salud, control y belleza a través de los medios de comunicación y la interacción social en general.

El problema que gira en torno a insatisfacción de la IC mediante la búsqueda del perfil corporal de delgadez, es que diversos estudios la han relacionado como uno de los factores claves que propicia el origen de trastornos de alimentación, como bulimia y anorexia nerviosa. Además esta insatisfacción se ha asocia a baja autoestima y depresión (Stice, 2001; Baile y cols., 2003; Polivy y Herman, 2002; Thompson, 2004), factores que no se midieron en este estudio, pero que son importantes relacionarlos con la insatisfacción de la IC para investigaciones posteriores en este campo.

Diversos estudios realizados en la actualidad, demuestran que los medios de comunicación masivos como la televisión pueden generar insatisfacción corporal (Levine y Harrison, 2004; Levine y cols., 2002), no obstante, para esta población la televisión no mostró influencia significativa sobre su insatisfacción corporal. Tampoco se encontró que el tener novio influya en la satisfacción corporal de estas adolescentes; en contraposición a los resultados de otras investigaciones que han encontrado que las relaciones sociales $y$ en especial las relaciones de pareja son un reforzador de los ideales corporales, al punto que pueden disminuir la insatisfacción y aumentar la autoestima (Whitehead y Hoover 2000; Lin y Kulik, 2002; Forbes y Cols., 2006). La única variable que se encontró que interviene en la satisfacción de la IC de esta población es el IMC, y específicamente a través del peso corporal. 
De acuerdo a los resultados se encontró que las adolescentes satisfechas de su IC presentaron un AF menor que las insatisfechas, pero esto no marcó diferencia significativa entre ambos grupos. No obstante, es importante mencionar que esta tendencia no coincide con la teoría que detalla que un desarrollo efectivo de la IC se acompaña de un mayor $\mathrm{AF}$, traducido al hecho de que el movimiento agudiza la percepción de la imagen personal, por que promueve el hecho de conocer lo que se puede y no se puede realizar, de acuerdo a la habilidad de las diferentes cualidades físicas que posee el sujeto (Fallas (2001).

En cambio se encontró que las adolescentes satisfechas con su IC presentaron mayor apariencia física (evaluada mediante el AF) que las insatisfechas, esto se presenta por la sencilla razón de que el primer grupo mencionado se acepta y se siente bien tal y como es, es decir, corporalmente es lo que quiere ser.

Otra relación significativa que arrojó el estudio fue que la apariencia física está relacionada de forma negativa con el IMC, por lo tanto, a menor IMC mayor apariencia física. Esto termina de corroborar que ésta población presenta una tendencia muy marcada hacia el "ideal de delgadez" que impone nuestra sociedad para sentirse bien y para ser aceptado, el cual es un factor detonante en comportamientos compulsivos de alimentación.

\section{CONCLUSIONES}

Las adolescentes estudiadas presentan una alteración del componente perceptual y cognitivo relacionado a su imagen corporal, traducido en una inadecuada percepción de su peso.

La satisfacción de la IC solo estuvo influenciada por el índice de masa corporal, sin ser relevante el papel que juegan los medios de comunicación y las relaciones personales de pareja en la muestra estudiada. Esta muestra de adolescentes persigue el "ideal de delgadez" que reportaron mediante la tendencia de que a menor índice de masa corporal tienen mayor satisfacción de la imagen corporal que poseen. En esta muestra, su satisfacción corporal no se encuentra orientada por la composición y dimensiones del cuerpo como tal sino por la percepción que tiene cada persona de la misma.

La recomendación más importante es realizar este mismo estudio pero a mayor escala en una muestra seleccionada aleatoriamente, para conocer si los resultados se repiten.

En investigaciones futuras se podría profundizar más estos hallazgos.

\section{REFERENCIAS}

Baile, J.I. (2000). ¿Qué es la imagen corporal?. Fuente: http://www.uned.es/catudela/revista/n002/baile ayensa.htm. sept 2006).

Baile, J. y Garrido, E. (s.f). Autoimagen referente al peso en un grupo de chicas adolescentes. Anales del sistema sanitario de Navarra. Fuente:

http://www.cfnavarra.es/salud/anales/textos/v ol22/n2/orig2a.html(24 sept 2006).

Baile, J., Raich, R., y Garrido, E. (2003). Evaluación de la insatisfacción corporal en adolescentes: efecto de administración de una escala. Anales de psicología, 10(2), 187-192.

Benninghoven, D., Eckhard, J., Mohr, A. Heberlein, I., Kunzendorf, S. y Jantschek, G. (2006). Different changes of body-images in pacients with anorexia or bulimia nervosa, during impatien psychosomatic treathment. European Eating Desorders Review, 14, 8896.

Cash, T. y Deagle, E. (1997). The natural and extent of body-image disturbances in anorexia nervosa and bulimia nervosa: a meta-analysis. International Journal of Eating Desorders, 22, 107-125.

Davison, T. y McCabe, M. (2006). Adolescent body image and psycholosocial functioning. Journal of Social Psychology, 146(1), 15-30.

Fallas, L. (2001). Autoconcepto general y Auticoncepto físico en personas físicamente activas $\mathrm{y}$ sedentarias en combinación con el género. Universidad Nacional. Heredia, Costa Rica.

Forbes, G., Jobe, R., y Richarson, R. (2006). Associations Between Having a Boyfriend and the Body Satisfaction and self-Esteem of college Women: An Extension of the Lin and Kulik Hypothesis. Journal of Social Psichology, 146 (3), 381-384. 
Groesz, L., Levine, M., y Murnen, S. (2002). The effect of experimental presentation of thin media images on body satisfaction: a meta-analytic review. International Journal of Eating Disorders, 31, 1-16.

Grogan, S. (1999). Body image: understanding body dissatisfaction in men, women and children. London: Routledge

Halliwell, E. y Dittmar, H. (2004). Does size matter? The impact of model's body size on advertising effectiveness and women's bodyfocused anxiety. Journal of Social and Clinical Psychology, 23, 105-132.

Hernández, R., Fernández, C., y Baptista, R. (2006). Metodología de la investigación. ( $\left.4^{\mathrm{a}} \mathrm{ed}\right)$. México, Distrito Federal: Mc Graw Hill.

Kerruish, K., Connor, J., y Hunphris, I. (2002). Body composition in adolescents with anorexia nervosa. Amerina Journal of Clinical Nutrition, 75, 31-7

Levine, M. y Harrison, K. (2004). The role of mass media in the perpetuation and preventin of negative body image and disordered eating. En Thompson, J. (2004). Handbook of Eating Disorders and Obesity. New York: Wiley.

Lin, L. y Kulik, J. (2002). Social Comparisons and women's body satisfaction. Basic and Applied Social Psychology, 24, 115-123.

Mahan, L. y Escott-Stump, S. (2000). Nutrición y Dietoterapia de Krause. $\left(10^{\mathrm{a}}\right.$ ed). México: McGraw-Hill.

Mayer, L., Walsh, T., Pearson, R., Heymsfield, S., Gallagher, D., Wang, J., Parides, M., Leibel, R., Warren, M., Killory, E., y Glasofer, D. (2005). Body fat redistribution after weight again in woman with anorexia nervosa. American Journal of Clinical Nutrition, 81, 1286-91.

Moncada, J. (2005). Estadística: para las ciencias del movimiento humano. San José, Costa Rica: Editorial de la Universidad de Costa Rica.

Polivy, J. y Herman, C. (2002). Causes of eating desorders. Anual Review of Psychology, 53, 187-213.

Probst, M., Morris, M., Vandereycken, W., y Van Coppenolle, H. (1996). Body composition in female anorexia nervosa patients. British Journal of Nutrition, 76, 639-47.

Organización Mundial de la Salud [OMS] (1993). El estado físico: Uso e interpretación de la antropometría (Serie de Informes Técnicos de un Comité de Expertos de la OMS 854). Ginebra, Suiza.

Rand, S. y Wright, B. (2000). Continuity and change in the evaluation of ideal and acceptable body sizes across a wide age span. International Journal of Eating Desorders. 28, 90-100.

Rowe, D., McDonald, S., Mahar, M., y Raedeke, T. (2005). Multitrait - multimethod investigation of a novel body image measurement technique. American Aliance for Health Physical Education, Recreation and Dance, 76(4), 407-415.

Stice, E. (2001). A prospective test of the dual-pathway model of bulimic pathaologic: mediating effects of dieting and negative effect. Journal of abnormal psychology, 110, 124-135.

Thompson, J. (2004). Handbook of Eating Disorders and Obesity. New York: Wiley.

Rodríguez, L. y Ulate, E. (2000). Manual de laboratorio en nutrición humana. San José, Costa Rica: Oficina de Publicaciones, Universidad de Costa Rica.

Whitehead, J. y Hoover, J. (2000). The link between body issues and behavioral problems. Reclaiming Children and Youth, 9, 130-132. 